\title{
Clinical Impact of Pelvic Malrotation on X-ray based Preoperative Planning for Total Hip Arthroplasty: A Proof-of-concept and Prudent Prediction of Acceptable Rotation
}

\section{Ernest Christian Lourens ( $\nabla$ ernestlourens@hotmail.com )}

LMH: Lyell McEwin Hospital https://orcid.org/0000-0002-7843-0225

\section{Andrew Kurmis}

Lyell McEwin Hospital

Wan Yin Lim

Royal Adelaide Hospital

\section{Research Article}

Keywords: X-ray, pelvic, rotation, hip, arthroplasty, preoperative planning, acetabular angle, computerised tomography

Posted Date: November 10th, 2021

DOI: https://doi.org/10.21203/rs.3.rs-1039052/v1

License: (c) (1) This work is licensed under a Creative Commons Attribution 4.0 International License.

Read Full License 


\section{Abstract}

\section{Introduction}

Pelvic rotation (PR) on preoperative X-ray templating can affect various critical measured acetabular angles and potentially outcomes of successful total hip arthroplasty (THA). Optimising anatomical reconstruction of the joint is essential to achieve function, longevity and prevention of complications following surgery. There is limited literature that standardises the degree of acceptable PR on X-ray or its effects on the fitting of acetabular prostheses.

\section{Objective}

This study aimed to develop a proof-of-concept that quantifies how PR can affect various acetabular angles used in pre-operative THA templating and to formulate a practicable method of determining if the preoperative PR is acceptable.

\section{Materials and Methods}

Computerised tomography (CT) models from three control and two THA patients were generated and manipulated in various degrees of PR. CT slices were thickened to simulate X-rays and acetabular angles measured.

\section{Results}

The acetabular anteversion distance (AAD) and lateral opening angle (LOA) demonstrated a linear and quadratic relationship with good correlation $\left(R^{2}=0.923, R^{2}=0.710\right.$ respectively, $\left.p<0.0001\right)$ in relation to PR. Change in area of prosthesis (AOP) demonstrated a good linear correlation $\left(r^{2}=0.774\right.$ and $r^{2}=0.875$, $\mathrm{p}<0.0001$ ) with PR. Two novel measurements were used to estimate the degree of PR from a pelvic X-ray; the horizontal distance between pubic symphysis and middle of sacrococcygeal joint (PSSC) and the simplified pelvic rotation ratio (SPRR). A strong correlation between PSSC and SPRR with change in PR was observed $\left(R^{2}=0.970, R^{2}=0.953, p<0.001\right)$.

\section{Conclusion}

Preliminary results suggests that an SPRR $>2.0$ correlates to $P R>20^{\circ}$ with potential to have a clinical impact on preoperative measurements.

\section{Background}

Total hip arthroplasty (THA) is a well-established method to treat hip pain and physical limitations due to osteoarthritis and other medical conditions. The acetabular prosthesis implantation angle affects muscle strength, gait, limb lengths, impingement, noise generation, loosening, postoperative range of movement (ROM) and is related to dislocation and liner wear [7]. Optimising biomechanical and anatomical 
reconstruction of the joint is essential to achieve function, longevity and prevention of complications following surgery [1-6]. Preoperative templating from a plain anterior-posterior radiograph is the primary method for initial evaluation and cornerstone for prosthesis positioning, however such images are subject to patient pelvic tilt and rotation. Suboptimal acetabular component position significantly impacts the results of a hip arthroplasty, including increased risk of instability, impingement, dislocation and cup failure [1, 3, 6, 8-13]. Correct template positioning influences the accuracy of acetabular cup placement planning and long-term success of the total hip arthroplasty [6].

Effects of pelvic position on pelvic tilt has been well studied [14-16]. When the pelvis tilts posteriorly, the anteversion and abduction angles of the acetabular implant increases, potentially leading to excessive wear due to neck impingement and edge loading, with increased risk of anterior dislocation [6]. Prediction of pelvic displacement before surgery is therefore essential for accurate placement of the acetabular implant.

Babisch et al. (2008) demonstrated that acetabular cup positions are affected by pelvic tilt on computerised tomography (CT) models, with good accuracy and reproducibility [17]. Similarly, a previous study used a computer-generated three-dimensional (3D) model to determine the effect of pelvic tilt on acetabular angles [18]. The functional angle of the acetabular implant is related to the pelvic tilt angle, with the anteversion angle of the acetabular implant changing by approximately 0.7 degrees with every degree in change in pelvic inclination.

In contrast, there is a lack of literature describing the effects of X-ray pelvic rotation (PR) on preoperative acetabular angulation of acetabular prostheses. The purpose of this study was to generate 3D CT pelvis models from healthy controls and arthroplasty patients and to quantify the potential effects of pelvic rotation on acetabular cup position in various planes. Furthermore, this study aimed to determine if there was a simple and practicable method of determining pelvic rotation on pre-operative templating. This proof-of-concept study hypothesised that PR may have detrimental impacts on acetabular angles during preoperative planning for THA. It also hypothesised that actual PR can be estimated from recreated plain X-rays using simple measurements and anatomical landmarks.

\section{Methods}

\section{Study Design:}

This was a retrospective case-control experimental design to investigate the effect of PR on acetabular angulation.

\section{Participants and setting:}

To establish a proof of concept, a convenience sample of control and patients undergoing total hip arthroplasty (THA) due to osteoarthritis from the Royal Adelaide Hospital was selected between the 1st January 2016 and 31st June 2018. In total five patient's pelvic CT images were analysed, with three 
controls and two THA patients. The control patient CT results were confirmed normal with no abnormalities by consultant radiologist prior to inclusion in the study. The THA CT images were also confirmed to be otherwise normal prior to inclusion. Baseline patient characteristics are recorded in Table 1. Definitions used to describe the angles and measurements used in this study can be found in Table 2 .

\section{Assessment of pelvic rotation using computerised model:}

True pelvic rotation was established using Carestream Picture Archiving and Communications Systems (PACS) and Radiology Information System (RIS) version 11. 0.14.35. For the acquisition of the images used in this study, thickened CT slices were used to simulate the X-ray radiographs in a 2-dimensional plane. The CT reconstruction was manipulated at varying degrees of rotation from the neutral position. The neutral PR was defined by ischiotuberosities being parallel to reference [19]. All pelvic images were aligned with a neutral pelvic tilt as described by Marratt et al. (2015) [18]. All parameters were measured on left hips. Pelvic rotation at increments of $10^{\circ}$ was performed up to $60^{\circ}$ in order to optimise interpretation data. Rotation was defined as positive $(+)$ rotation corresponding to pelvic rotation to the left (relative pelvic External Rotation, ER) and negative (-) rotation corresponding to pelvic rotation to the right (Internal rotation, IR). At each $10^{\circ}$ increment, the AP and lateral 2-dimensional XR images of the pelvis was recorded. Respective angles, measurements and ratio calculations were undertaken using the definitions summarised in Table 2.

\section{Pelvic rotation (PR):}

PR on AP X-ray was estimated using the horizontal distance between the pubic symphysis and middle of the sacrococcygeal joint (PSSC) using the methods described in Tannast et al. (2005) [20]. We proposed a refined method for PR assessment using the ratio between the PSSC and the horizontal distance between the two anterior superior iliac spines (HASIS), thus allowing a more patient specific determination. We named this measurement the simplified pelvic rotation ratio (SPRR).

\section{Statistical analysis:}

Statistical analysis was performed with the Statistical Package for the Social Sciences (SPSS) for Windows, Version 24.0, IBM Corp. Released 2016. Armonk, NY. A highly precise value of $p<0.001$ was accepted as indicating statistical significance. Data was expressed as mean \pm standard deviation for continuous variables and as a number and percentage for categorical variables. Categorical data between the groups was compared using the Chi-squared test or Fisher's exact test, while continuously distributed data was compared using either a Mann-Whitney $U$ test or an analysis of variance (ANOVA). Linear and quadratic trend lines were generated using SPSS and reported using a R-squared value. Line goodness of fit was assessed using an ANOVA linear or quadratic regression and reported using an Fvalue with a significance level of $p<0.001$ used.

\section{Results}




\section{Acetabular measurements:}

There were no statistically significant differences in the baseline characteristics of the control hips and THA groups used to generate CT models (Table 1).

\begin{tabular}{|llll|}
\hline \multicolumn{4}{|c|}{ Table 1 } \\
Baseline characteristics of participating patients \\
& Control & THA & Sig. $(\mathrm{p}=)$ \\
\hline Patient demographics & & \\
\hline Number of participants: (M/F) & $3(3 / 0)$ & $2(0 / 2)$ & 0.1 \\
\hline Age years: mean (SD) & $45(31.19)$ & $58(28.28)$ & 0.699 \\
\hline Past medical history of: (n) & & & \\
\hline Hip Osteo Arthritis & 1 & 2 & 0.400 \\
\hline Osteoporosis & 0 & 1 & 0.400 \\
\hline T2DM & 0 & 1 & 0.400 \\
\hline Heart Failure & 0 & 1 & 0.400 \\
\hline Corticosteroid use & 0 & 1 & 0.400 \\
\hline & & & 1.0 \\
\hline THA side (L:R) & $(0: 1)$ & $(2: 1)$ & \\
\hline
\end{tabular}

Table 3 describes the mathematic trend relationship of best fit between PR, the aforementioned hip angles and measurements of interest, with all trends calculated to be statistically significant $(p<0.001)$. 
Table 3

Effect of pelvic rotation on various measurements

Native THR Trend F-value $\mathrm{R}^{2}=\underset{\substack{\text { Sig. } \\(\mathrm{p}=)}}{\text { Graph }}$

\section{Acetabular Measurement}

\begin{tabular}{|c|c|c|c|c|c|c|c|}
\hline $\begin{array}{l}\text { Acetabular anteversion } \\
\text { distance (AAD) }\end{array}$ & $n=3$ & $\mathrm{n}=0$ & Linear & 394.65 & 0.923 & $<0.001$ & 1 \\
\hline $\begin{array}{l}\text { Acetabular inclination angle } \\
\text { (AIA) }\end{array}$ & $n=3$ & $\mathrm{n}=0$ & Linear & 18.42 & 0.332 & $<0.001$ & - \\
\hline $\begin{array}{l}\text { Acetabular inclination angle } \\
\text { (AIA) }\end{array}$ & $n=3$ & $n=2$ & Linear & 20.89 & 0.359 & $<0.001$ & 2 \\
\hline Lateral opening angle (LOA) & $n=3$ & $\mathrm{n}=0$ & Quadratic & 60.75 & 0.771 & $<0.001$ & - \\
\hline Lateral opening angle (LOA) & $n=3$ & $\mathrm{n}=2$ & Quadratic & 75.98 & 0.710 & $<0.001$ & 3 \\
\hline Area of prosthesis (AOP) & $\mathrm{n}=0$ & $\mathrm{n}=2$ & Linear & 19.56 & 0.449 & $<0.001$ & 4 \\
\hline Acetabular diameter (AD) & $n=3$ & $\mathrm{n}=0$ & Quadratic & 8.9 & 0.313 & $<0.001$ & - \\
\hline \multicolumn{8}{|l|}{ Estimation of rotation } \\
\hline PSSC & $n=3$ & $\mathrm{n}=0$ & Linear & 884.39 & 0.960 & $<0.001$ & - \\
\hline PSSC & $n=3$ & $n=2$ & Linear & 2016.55 & 0.970 & $<0.001$ & 5 \\
\hline SPRR & $n=3$ & $\mathrm{n}=0$ & Linear & 502.32 & 0.931 & $<0.001$ & - \\
\hline SPRR & $n=3$ & $n=2$ & Linear & 1284.32 & 0.953 & $<0.001$ & 6 \\
\hline
\end{tabular}

The acetabular anteversion distance (AAD) and lateral opening angle (LOA) demonstrated a linear and quadratic relationship with good correlation ( $R 2=0.923, R 2=0.710$ respectively) in relation to pelvic rotation (Figure A, Graphs 1 and 3).

Combined change in area of prosthesis $(\mathrm{AOP})$ demonstrated a moderate $(\mathrm{R} 2=0.449)$ linear correlation with pelvic rotation (Figure A Graph 4).

This does not however account for different implant sizes. If separated into two analyses, prosthesis 1 and 2 yielded the following respectively, $r 2=0.774, p<0.001, F=37.67$, and $r 2=0.875, p<0.001, F=77.27$.

Change in acetabular inclination angle (AIA) and acetabular diameter (AD) both demonstrated weak correlations, with only approximately a third of the change variance explained by pelvic rotation using our model. 
Change in PSSC and SPRR demonstrated very strong linear correlations with pelvic rotation respectively (R2=0.970, R2=0.953, $p<0.001$, graph 5 and 6).

\section{Discussion}

The AP radiograph remains the mainstay initial evaluation of pelvic anatomy for preoperative THA planning. Perceived pelvic anatomy and associated angles and measurements on an AP radiograph depend directly on the extent of PR. This study aimed to determine the relationship between PR and such measurements, if there is there an acceptable degree of $P R$, and if there is there a simple method of quantifying PR from an AP pelvic radiograph. Using the CT reconstructed images, the degree of pelvic rotation was able to be manipulated and recorded with high precision. By neutralising pelvic tilt completely, the potential confounding effect of pelvic tilt on acetabular angles are ameliorated.

For the purposes of this discussion in the context of interpretation in preoperative THA planning, trendlines of Graphs 1-6 (Figure 1) where analysed and approximations were made between the angles of 0 to $40^{\circ}$ relative pelvic internal rotation (IR) and external rotation (ER). We recognise that there are number of different methods to assess acetabular anteversion in native and THA patients [21]. Our data suggests that the observed AAD (Graph 1 ) increases linearly by approximately $2-3^{\circ}$ for every $4^{\circ}$ of pelvic rotation in either direction. It is clinically necessary to ensure an appropriately neutral pelvis to avoid falsely interpreting excessive anteversion. We suggest that up to $20^{\circ}$ of PR will lead to a safe estimation of AAD.

In contrast, the lateral opening angle (LOA) tends to increase linearly from relative pelvic IR to ER by approximately $1^{\circ}$ for every $4^{\circ}$ of PR. Rojas et al. (2018) [22] found that an altered pre-operative Sharp's angle (methodologically similar to LOA [23] ) was associated with cup malpositioning (OR 2.51, $\mathrm{p}=0.02$ ). It is therefore imperative to ensure minimal PR and therefore accurate preoperative LOA to avoid potential cup malpositioning and therefore potentially poor THA outcomes. There is a sparsity of literature reporting the effect of PR on LOA, however our results are consistent with one study's conclusion that PR increases the error of acetabular index in 3-month olds with developmental dysplasia of the hip [24]. A study from Tannast et al. (2015) [19] that applied a similar method using CT imaging demonstrated methodological accuracy with good reproducibility and reliability. It also revealed Sharp's angle did not have a clinically significant change between neutral to $12^{\circ}$ (bilaterally), however their experiment did not report beyond $12^{\circ}$ of PR. Clinically we suggest that up to $20^{\circ}$ of PR will lead to minimal impact on the final measurement of LOA.

The AOP tends to increase linearly from relative pelvic ER to IR by approximately $1 \mathrm{~cm}^{2}$ for every $4^{\circ}$ of PR. The AOP for the two different implant sizes in in Graph 4 must be analysed separately in order to be clinically meaningful, yielding good linear correlation with PR. This has the potential clinical implication that a relative pelvic internal rotation can result in an under estimation of post-operative acetabular prosthetic coverage and therefore implant size choice. Likewise, PR of less than $20^{\circ}$ is unlikely to pose a significant negative impact on AOP estimation. It is also recommended that future analyses group datapoints for similar implant sizes to reduce unnecessary variation. 
Taking into consideration that both AIA and AD demonstrated a weak correlation with PR, the AIA tends to increase linearly from relative pelvic ER to IR by approximately $1^{\circ}$ for every $5^{\circ}$ of $P R$. AD tended to follow a quadratic trendline, where both IR and ER of the pelvis reduced observed $A D$ by approximately $1.5 \mathrm{~mm}$ per $10^{\circ}$ of rotation. A study by Ghostine et al. (2016)[25] investigating 3-D reconstructions from biplanarradiographs also reported that AIA and acetabular anteversion was minimally affected by PR from neutral to $20^{\circ}$ in comparison with other hip parameters, thereby supporting our results. The clinical applicability of our AIA and AD data is however tenuous.

As previously discussed, incorrect acetabular prosthetic cup positioning is known to be associated with poor clinical outcomes, such as impingement, instability or prosthetic failure $[1,4,2,3,5]$. It is therefore important to determine an acceptable degree to PR that will not significantly bias preoperative planning. Synthesising results from this proof-of-concept study, PR of more than $20^{\circ}$ can produce unreliable acetabular angles and measurements, leading to increased risk of incorrect prosthesis placement. It is therefore imperative to ensure a safe amount of PR on AP radiograph.

\section{Estimation of rotation:}

In this study, actual pelvic rotation determined by CT can be estimated on AP X-ray using the horizontal distance between the pubic symphysis and middle of the sacrococcygeal joint (PSSC) and the horizontal distance between the two anterior superior iliac spines (HASIS). The results show a near-perfect correlation between PSSC and PR, giving a clear indication to the extent of PR on an AP radiograph. These results are comparable to a previous study [20] that employed similar methodology on 20 cadavers. Using the trendline from Graph 5 , a PSSC of less than $10 \mathrm{~mm}$ is expected at $0^{\circ}$ of pelvic rotation, with an increase of approximately $20 \mathrm{~mm}$ for every $10^{\circ}$ of $\mathrm{PR}$ in either direction.

It can be argued however that these measurements are subject to individual patient anthropometry, gender, size, radiographic technique and analysis software, therefore a more patient-specific method was investigated. By using a novel ratio, the SPRR, patient factors and imaging techniques were expected to have less overall impact. Using Graph 6, at neutral pelvic rotation the SPRR is shown to be less than 1 . As PR increases in either direction, PSSC (measured in $\mathrm{mm}$ ) increases and HASIS (measured in $\mathrm{cm}$ ) decreases, therefore the ratio increases. Analysing preliminary data used in this study, an SPRR greater than 2.0 is therefore likely associated with X-ray pelvic rotation of more than $20^{\circ}$. It would therefore seem appropriate for this value to be employed as a safe cut off for PR tolerance.

\section{Limitations:}

This novel proof-of-concept study does have some methodological limitation, including a limited sample size of pelvises, single observer and unilateral hip measurements. It would therefore be questionable to generalise the exact values to a particular age bracket, gender or specific diagnostic indication for THA. The 3-D pelvis models generated we used was based on real human pelvises, therefore are subject to individual anatomical differences. The goal of this study however was not to necessarily to report the 
absolute acetabular angles, but rather to demonstrate the effect of PR on these values. We reasoned that pelvises of differing anatomical structure would have similar degrees of changes due to PR, regardless of the absolute acetabular angle measurement. Despite these limitations therefore, the trendlines discussed demonstrated acceptable goodness of fit and clinically useful data for, AAD, LOA, estimation of rotation using PSSC and SPRR. Based on these outcomes we were able to provide an estimate the potential acceptableness of PR from an AP pelvis radiograph.

\section{Conclusion}

Pelvic rotation can significantly impact on the perceived acetabular angles observed on an AP pelvic radiograph, which can in turn result in poor prosthetic placement and potentially subsequent poorer longterm clinical outcomes. Data from our initial study indicates that PR of less than $20^{\circ}$ is unlikely to have a clinical impact of preoperative measurements and therefore serve as a guide for clinical application.

We propose that our new and novel ratio of SPRR of more than 2.0 may serve as a simple and reproducible surrogate for measured PR of $>20^{\circ}$.

\section{Abbreviations}

3D: Three-dimensional

AAD: Acetabular anteversion distance

AD: Acetabular diameter

AIA: Acetabular inclination angle

ANOVA: Analysis of variance

AOP: Area of prosthesis

CT: Computerised tomography

ER: External rotation

HASIS: horizontal distance between the two anterior superior iliac spines

IR: Internal rotation

LOA: Lateral opening angle

PACS: Picture archiving and communications systems

PR: Pelvic rotation 
PSSC: Horizontal distance between pubic symphysis and middle of sacrococcygeal joint

RIS: Radiology information system

ROM: Range of movement

SPRR: Simplified pelvic rotation ratio

SPSS: Statistical package for the social sciences

THA: Total hip arthroplasty

\section{Declarations}

\section{Ethics approval and consent to participate}

Ethics approval was granted by the Central Adelaide Local Health Network Human Research Ethics Committee (Level 3, Roma Mitchell House 136 North Terrace Adelaide, South Australia, 5000 Telephone: +61 87117 2229) on 19/12/2018. HREC reference number: HREC/18/CALHN/686. CALHN Reference number: R20181004.

The approval letter is attached in a separate document.

\section{Consent for publication}

Not applicable

\section{Availability of data and materials}

The datasets used and/or analysed during the current study are available from the corresponding author on reasonable request.

\section{Competing interests}

Not applicable

\section{Funding}

Not applicable 


\section{Acknowledgements}

Not applicable

\section{Authors' Information and Contribution}

First Author and Corresponding Author: Dr Ernest Lourens

MD (Dist.), B. Physio (Hons), GradCertClinRehab

Lyell McEwin Hospital, Haydown Road Elizabeth Vale SA 5112

Email: Ernest.Lourens@sa.gov.au, ernestlourens@hotmail.com

Author contribution statement: Author contribution statement: This author has contributed to this research (1) conception and design, or acquisition of data, or analysis and interpretation of data, (2) drafting the article or revising it critically for important intellectual content, (3) final approval of the version to be published, and (4) agree to be accountable for all aspects of the work if questions arise related to its accuracy or integrity. The authors declare that they have no conflict of interest.

Associate Professor Andrew P Kurmis

FRACS (Ortho), FAOrthA, CIME, PhD (Ortho), BMBS (Hons), BMedRad (Hons), BAppSc (Med Rad)

Lyell McEwin Hospital, Haydown Road Elizabeth Vale SA 5112

Email: Andrew.Kurmis@sa.gov.au

Author contribution statement: This author has contributed to this research (1) conception and design, or acquisition of data, or analysis and interpretation of data, (2) drafting the article or revising it critically for important intellectual content, (3) final approval of the version to be published, and (4) agree to be accountable for all aspects of the work if questions arise related to its accuracy or integrity. The authors declare that they have no conflict of interest.

Dr Lim Wan Yin,

MBChB, FRANZCR

Royal Adelaide Hospital, Port Rd, Adelaide SA 5000

Email: Wanyin.lim@sa.gov.au

Author contribution statement: Author contribution statement: This author has contributed to this research (1) conception and design, or acquisition of data, or analysis and interpretation of data, (2) 
drafting the article or revising it critically for important intellectual content, (3) final approval of the version to be published, and (4) agree to be accountable for all aspects of the work if questions arise related to its accuracy or integrity. The authors declare that they have no conflict of interest.

\section{References}

1. Meftah M, Yadav A, Wong AC, Ranawat AS, Ranawat CS. A novel method for accurate and reproducible functional cup positioning in total hip arthroplasty. The Journal of arthroplasty. 2013 Aug 1;28(7):1200-5.

2. Ng VY, McShane MA. Understanding acetabular cup orientation: The importance of convention and defining the safe zone. Hip International. 2011 Nov;21(6):646-52.

3. Merle C, Grammatopoulos G, Waldstein W, Pegg E, Pandit H, Aldinger PR, Gill HS, Murray DW. Comparison of native anatomy with recommended safe component orientation in total hip arthroplasty for primary osteoarthritis. The Journal of Bone \& Joint Surgery. $2013 \mathrm{Nov}$ 20;95(22):e172.

4. McBride A, Flynn J, Miller G, Barnes M, Mackie S. Body mass index and acetabular component position in total hip arthroplasty. ANZ journal of surgery. 2013 Mar;83(3):171-4.

5. Grammatopoulos, G., Thomas, G., Pandit, H., Beard, D., Gill, H. and Murray, D., 2015. The effect of orientation of the acetabular component on outcome following total hip arthroplasty with small diameter hard-on-soft bearings. The Bone \& Joint Journal, 97-B(2), pp.164-172.

6. Bhaskar D, Rajpura A, Board T. Current concepts in acetabular positioning in total hip arthroplasty. Indian journal of orthopaedics. 2017 Aug;51(4):386-96.

7. Scheerlinck T. Cup positioning in total hip arthroplasty. Acta Orthopaedica Belgica. 2014 Sep 1;80(3):336-47.

8. Barrack RL, Krempec JA, Clohisy JC, McDonald DJ, Ricci WM, Ruh EL, Nunley RM. Accuracy of acetabular component position in hip arthroplasty. The Journal of Bone \& Joint Surgery. 2013 Oct 2;95(19):1760-8.

9. Domb BG, El Bitar YF, Sadik AY, Stake CE, Botser IB. Comparison of robotic-assisted and conventional acetabular cup placement in THA: a matched-pair controlled study. Clinical Orthopaedics and Related Research. 2014 Jan;472(1):329-36.

10. Korduba LA, Essner A, Pivec R, Lancin P, Mont MA, Wang A, Delanois RE. Effect of acetabular cup abduction angle on wear of ultrahigh-molecular-weight polyethylene in hip simulator testing. The American Journal of Orthopedics. 2014 Oct;43(10):466-71.

11. Lewinnek GE, Lewis JL, Tarr RI, Compere CL, Zimmerman JR. Dislocations after total hip-replacement arthroplasties. Journal of Bone Joint Surgery. 1978 Mar 1;60(2):217-20.

12. Barrack RL. Dislocation after total hip arthroplasty: implant design and orientation. JAAOS-Journal of the American Academy of Orthopaedic Surgeons. 2003 Mar 1;11(2):89-99. 
13. Callanan MC, Jarrett B, Bragdon CR, Zurakowski D, Rubash HE, Freiberg AA, Malchau H. The John Charnley Award: risk factors for cup malpositioning: quality improvement through a joint registry at a tertiary hospital. Clinical Orthopaedics and Related Research. 2011 Feb;469(2):319-29.

14. Mellano CR, Spitzer Al. How does pelvic rotation or tilt affect radiographic measurement of acetabular component inclination angle during THA?. Journal of orthopaedics. $2015 \mathrm{Dec}$ 1;12(4):222-7.

15. Kyo T, Nakahara I, Miki H. Factors predicting change in pelvic posterior tilt after THA. Orthopedics. 2013 Jun 1;36(6):e753-9.

16. Lembeck B, Mueller $\mathrm{O}$, Reize P, Wuelker N. Pelvic tilt makes acetabular cup navigation inaccurate. Acta orthopaedica. 2005 Jan 1;76(4):517-23.

17. Babisch JW, Layher F, Amiot LP. The rationale for tilt-adjusted acetabular cup navigation. The Journal of Bone \& Joint Surgery. 2008 Feb 1;90(2):357-65.

18. Maratt JD, Esposito Cl, McLawhorn AS, Jerabek SA, Padgett DE, Mayman DJ. Pelvic tilt in patients undergoing total hip arthroplasty: when does it matter?. The Journal of arthroplasty. 2015 Mar 1;30(3):387-91.

19. Tannast M, Fritsch S, Zheng G, Siebenrock KA, Steppacher SD. Which radiographic hip parameters do not have to be corrected for pelvic rotation and tilt?. Clinical Orthopaedics and Related Research. 2015 Apr;473(4):1255-66.

20. Tannast M, Zheng G, Anderegg C, Burckhardt K, Langlotz F, Ganz R, Siebenrock KA. Tilt and rotation correction of acetabular version on pelvic radiographs. Clinical Orthopaedics and Related Research (1976-2007). 2005 Sep 1;438:182-90.

21. Park YS, Shin WC, Lee SM, Kwak SH, Bae JY, Suh KT. The best method for evaluating anteversion of the acetabular component after total hip arthroplasty on plain radiographs. Journal of orthopaedic surgery and research. 2018 Dec;13(1):1-8.

22. Rojas J, Bautista M, Bonilla G, Amado O, Huerfano E, Monsalvo D, Llinás A, Navas J. A retrospective study on the relationship between altered native acetabular angle and vertical implant malpositioning. International orthopaedics. 2018 Apr;42(4):769-75.

23. Sharp IK. Acetabular dysplasia: the acetabular angle. The Journal of Bone and Joint Surgery. British Volume. 1961 May;43(2):268-72.

24. Van der Bom MJ, Groote ME, Vincken KL, Beek FJ, Bartels LW. Pelvic rotation and tilt can cause misinterpretation of the acetabular index measured on radiographs. Clinical Orthopaedics and Related Research. 2011 Jun;469(6):1743-9.

25. Ghostine B, Sauret C, Assi A, Bakouny Z, Khalil N, Skalli W, Ghanem I. Influence of patient axial malpositioning on the trueness and precision of pelvic parameters obtained from 3D reconstructions based on biplanar radiographs. European radiology. 2017 Mar 1;27(3):1295-302.

\section{Tables}


Due to technical limitations, table 2 is only available as a download in the Supplemental Files section.

\section{Figures}
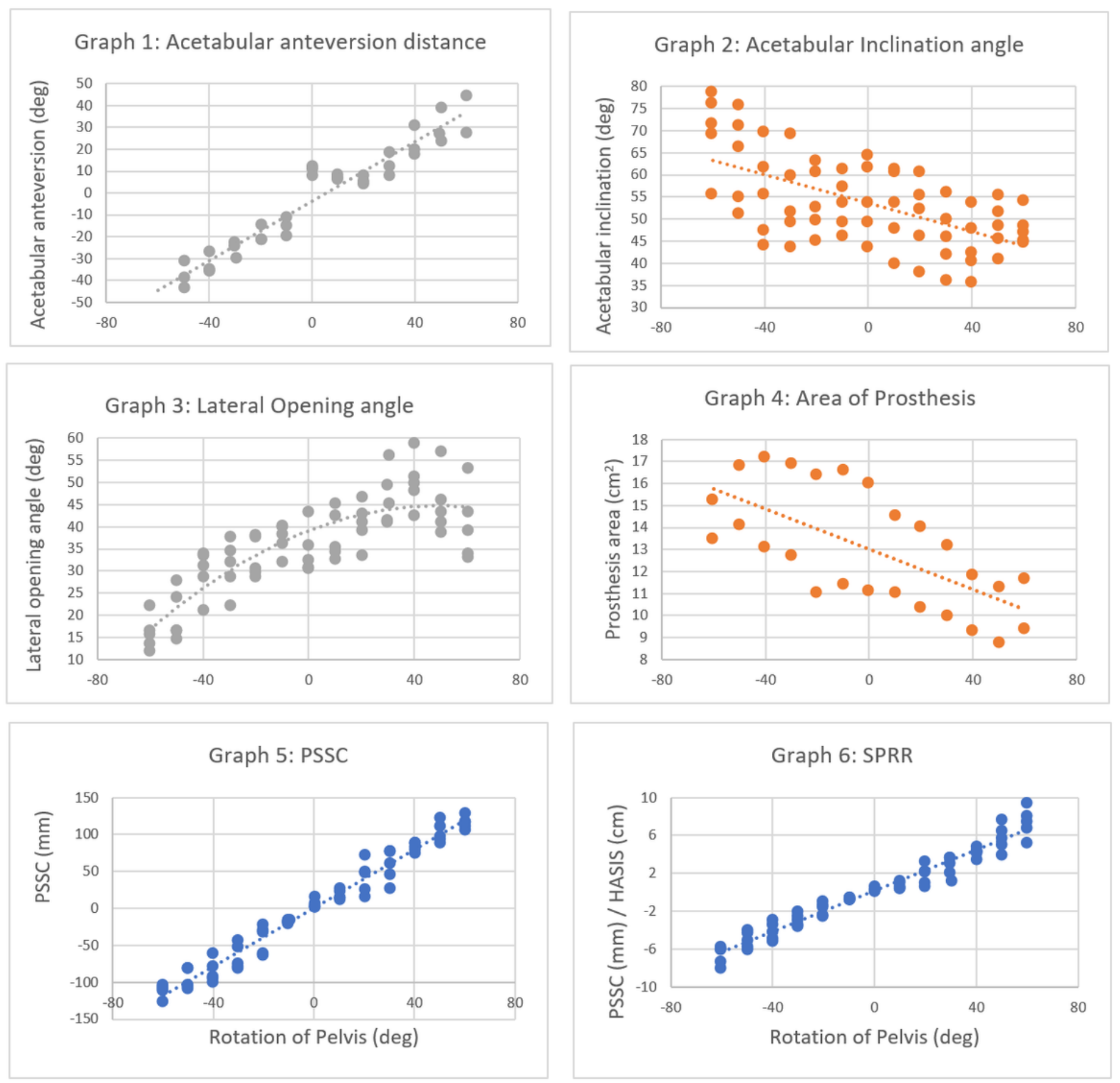

\section{Figure 1}

Graphs demonstrating effect of pelvic rotation on various angles. Figure Legend: All observations made on left hips. Positive (+) rotation corresponds to pelvic rotation to the left (i.e. relative pelvic External Rotation, ER), negative (-) rotation corresponds to pelvic rotation to the right (Internal rotation, IR). 


\section{Supplementary Files}

This is a list of supplementary files associated with this preprint. Click to download.

- Table2.docx 